\title{
Reorganizing A New Generation Airline Network Based on An Ant-Colony Optimization-Inspired Small-World Network
}

\author{
Hidefumi Sawai \\ Advanced ICT Research Institute, National Institute of Information and Communications Technology \\ Kobe 651-2492 Japan, Email: sawai@nict.go.jp
}

\begin{abstract}
We describe a real-world application for a next generation airline network using a novel Small-World (SW) network architecture. An emergent method that creates a new type of Small-World network with less average path-length than that obtained with conventional small-world networks is presented. This method is inspired from an Ant-Colony Optimization (ACO) algorithm. The resultant network architecture becomes a multistar network, which yields a large clustering coefficient and the shortest average path-length among the conventional complex networks such as the Watts-Strogatz and Barabási-Albert models etc., from both a theoretical and an experimental analysis of the properties of those networks. Given the advantageous properties of the multi-star network in real-world applications, it could be used to design a new generation global airline network superseding in terms of efficiency and convienience the current, conventional airline network owing to fewer transits and a shorter cruising distance on average from any starting point to any destination on Earth. This will be beneficial not only both to travelers and airline companies, but will also contribute to the reduction of greenhouse gases such as carbon dioxide $\left(\mathrm{CO}_{2}\right)$ in the near future, while enhancing communications and transportation worldwide.
\end{abstract}

Keywords-Ant-Colony Optimization (ACO), Small-World, airline networks, n-star networks, complex networks, average path length

\section{INTRODUCTION}

\section{A. Conventional Airline Networks}

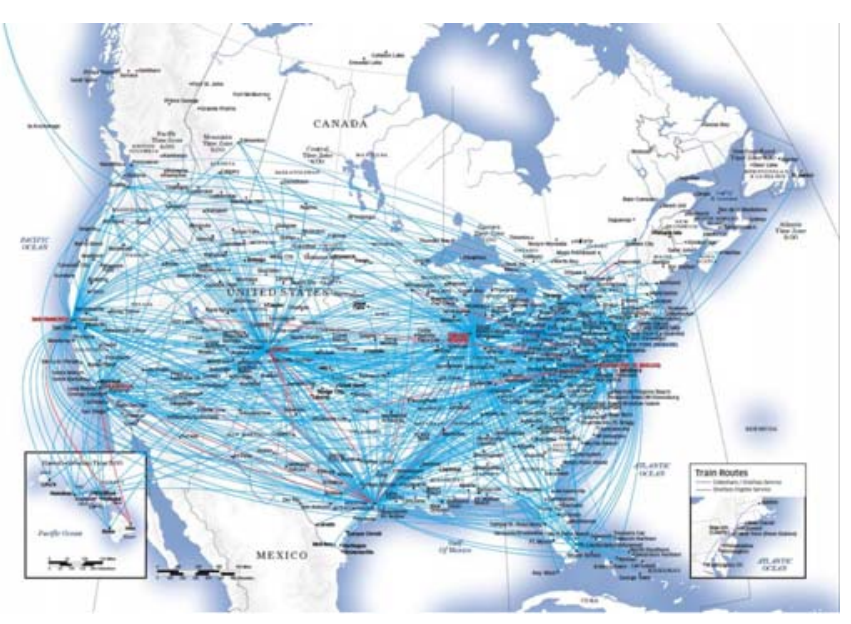

Fig. 1. A conventional airline network in North America.[1]

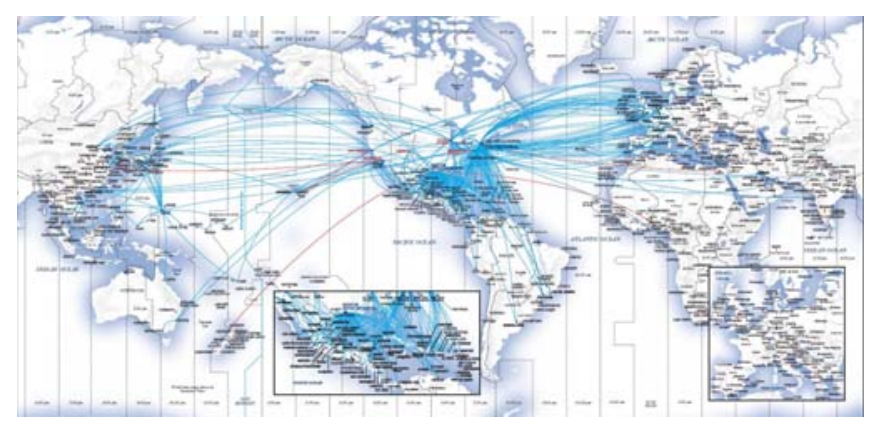

Fig. 2. A conventional global airline network.[2]

Typical conventional airline networks are shown in Fig.1 and Fig. 2 in the cases of a North America and of a global airline network, respectively. A significant characteristic of these network architectures is that they are called "scale-free", which means that more links tend to connect to a node with more degree (the connection probability is proportional to the number of links possessed by the node). This is called "preferential attachment". The scale-free networks are often modeled by a Barabási-Albert (BA) model [5]. This kind of airline network has so far been considered to be convenient for both travelers and airline companies because people can reach any destination from any starting city if the corresponding flight between the two cities is available. However, have the current airline networks been optimized in terms of the efficiency of the cruising distance (i.e., saving jet fuel) and of convenience for travelers (i.e., saving cruising time and minimizing the number of transits) as a whole? Isn't there any room to increase both the efficiency and convenience by changing the architecture or topology of the existing airline networks?

Very recently, a next-generation airplane, the Boeing 787, has been brought into in service [10]. This airplane is middlesized and has good characteristics that are designed to ensure energy-saving. It can operate a non-stop flight over a distance of $14,200-15,200 \mathrm{~km}$ (this distance corresponds to a flight between Tokyo in Japan and Johannesburg in South Africa) because of its light body and energy efficiency. Many airline companies have already purchased and/or ordered a number of models of this airplane [10]. Changing the current airline 
networks to design more efficient and convenient networks using this airplane will be greatly beneficial for both flying and travelers in terms of reducing jet fuel consumption and travel time because of shorter cruising distances and transit times. To achieve this goal, we explore a novel Small-World network inspired from Ant-Colony Optimization (ACO) [8], whose average path length is shorter than that obtained with any other conventional complex network, such as a WattsStrogatz model [4] and a Barabási-Albert model [5] etc. Thus, travel time and jet fuel consumption of airplanes are reduced by cruising on the shortest path between any starting city and destination on the Earth, which not only increases fuel efficiency (i.e., saves energy) and reduces both travel times and the number of transits (to the benefit of passengers), but also reduces the emission of greenhouse gases such as carbon dioxide $\left(\mathrm{CO}_{2}\right)$ on Earth, while enhancing communications and transportation worldwide.

\section{B. Background of the Small-World}

In the 1960's, S.Milgram performed a famous Small-world experiment using letter delivery in the US [3]. From this study, he coined the term "Six-degrees of separation", which means that people are separated from only six persons on average. Later, several researchers additionally performed similar experiments for various kinds of communities such as actors in Hollywood, co-authors of mathematical papers, etc. [4], which supported the claim made by Milgram based on his experiment. Recently, Yahoo began a similar experiment using Facebook to verify the "Six-degrees of separation" hypothesis for the members of Facebook [6].

In this study, we will re-examine the characteristics of the Small-World using several network parameters, such as the number of nodes $N$, the average degree $\langle k\rangle$, the average path-length $L$, the clustering coefficient $C$, the link exchange probability $\rho$, etc., through experimental simulations using the Dijkstra method [9], searching for the shortest path in several complex networks.

Generally speaking, it is said that the WS (Watts-Strogatz) and BA (Barabási-Albert) models yield comparatively shorter average path-lengths than those of the other complex networks. However, we will further try to have new Small-World networks emerge with a shorter average path-length (it might be the shortest one) in a self-organizing manner.

Next, we will analyze the resultant network architecture theoretically and experimentally. By defining a new network index $R$, we will show that the degree of the Small-World (we call it "Small-Worldness") can be evaluated for several complex networks. Finally, we will show that this new smallworld network can be applied to a new generation airline network in the real world.

\section{Comparison of Complex Networks}

In Table 1, we summarize the features of conventional complex networks in terms of the Small-World using several network indexes. Also, in Fig.3, we show some typical examples of complex networks, such as a random graph, the BA
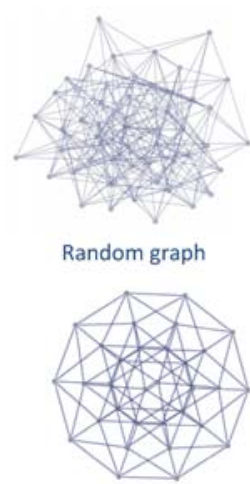

Hypercube $(D=5)$
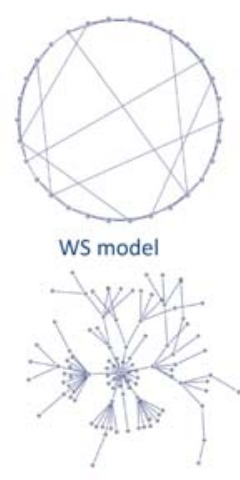

Tree
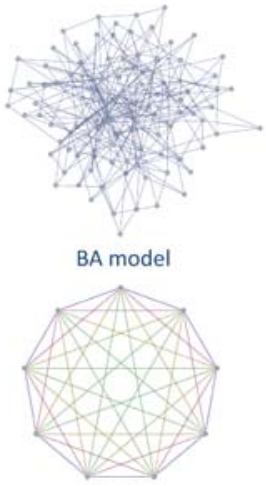

Complete graph
Fig. 3. Typical Complex Networks

Table 1. Comparing characteristics in typical complex networks. [7]

\begin{tabular}{|c|c|c|c|}
\hline Network & k (degree) & $\begin{array}{l}\text { L (average path- } \\
\text { length) }\end{array}$ & $\begin{array}{l}\text { C(Clustering } \\
\text { coefficient) }\end{array}$ \\
\hline Real Network & $\begin{array}{l}\text { power law } \\
\propto k^{v} \text { etc. }\end{array}$ & small $O(\log N)$ & large $O(1)$ \\
\hline Complete graph & $k=N-1$ & small 1 & large 1 \\
\hline $\begin{array}{l}\text { Lattice and cycle } \\
\text { (dimension: D) }\end{array}$ & uniform & large $O\left(N^{1 / D}\right)$ & large $O(1)$ \\
\hline Tree & uniform & small $O(\log N)$ & small 0 \\
\hline Random graph & $\begin{array}{l}\text { Poisson } \\
\text { distribution }\end{array}$ & small $O(\log N)$ & small $O(1 / N)$ \\
\hline $\begin{array}{l}\text { Watts-Strogatz } \\
\text { model }\end{array}$ & uniform & $\begin{aligned} N /(2<k>) ; & N<k>p / 2<<1 \\
\log (N<k>p) /(<k>p) ; & \\
N & <k>p / 2 \gg 1\end{aligned}$ & $(3<k>-6)(1-p)^{3} /(4<k>-4)$ \\
\hline $\begin{array}{l}\text { Barabási-Albert } \\
\text { model }\end{array}$ & $\begin{array}{l}\text { power law } \\
\propto k^{3} \text { etc. }\end{array}$ & $\begin{array}{l}\text { small O(logN); } \quad m=1 \\
O(\log N / \log \log N) ; m \geqq 2\end{array}$ & $(m-1)(\log N)^{2} / 8 N$ \\
\hline $\begin{array}{l}\text { Hypercube } \\
\text { (dimension: D) }\end{array}$ & uniform: $D$ & $\begin{array}{c}\text { small } O(\log N)=O\left(\log 2^{D}\right) \\
=O(D)=D 2^{(D-1)} /\left(2^{D}-1\right)\end{array}$ & small 0 \\
\hline
\end{tabular}

(Barabási-Albert) model, the Tree, the WS (Watts-Strogatz) model [4], the hypercube and the complete graph. Following are some network indexes used in this paper;

- Number of nodes: $N$.

- Average degree: $\langle k\rangle$.

- Link probability: $p=\langle k\rangle /(N-1)$.

- Average path length: $L$.

- Clustering coefficient: $C$.

- Link exchange probability: $\rho$ (in the case of the WS model).

Comparing the average path-lengths among the complex networks in Table 1 , the order of the average path-length $L$ is $O(\log N)$ for the Tree, random graphs, BA models and hypercubes as well as for the real networks, which is comparatively shorter than for other kinds of networks. The clustering coefficient $C$ is large for real networks, lattices and cycles, but small for Trees, random graphs and hypercubes. For a WS model, starting from an extended cycle, as the link exchange probability $\rho$ gradually increases from 0 , it is well known that the average path length dramatically decreases in the range 
Sorting all edges in the order of pheromone concentration
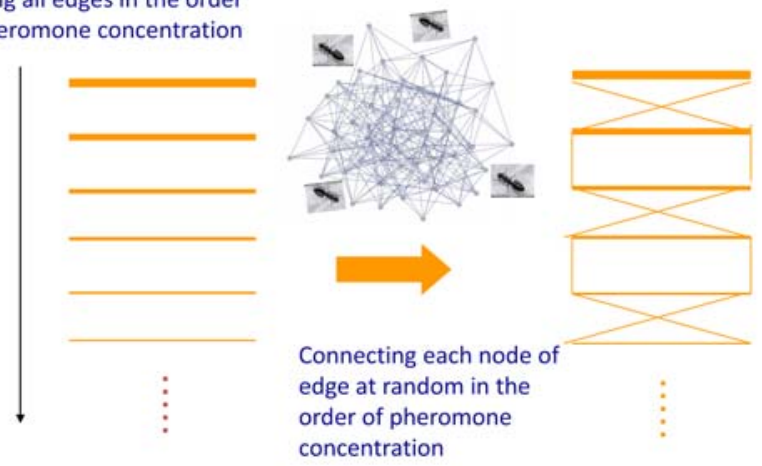

Fig. 4. Sorting the order of pheromone concentration on links along the shortest path.
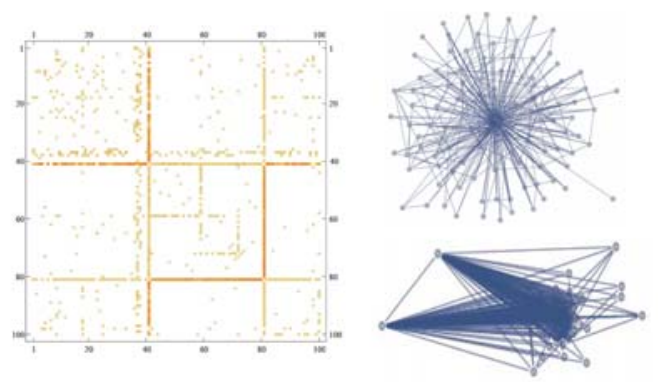

Fig. 5. Adjacency Matrix (left) and its corresponding network (right) generated by a method inspired from ACO (Pseudo-Ant-SW Method) when $N=100$.

of small values of $\rho$. However, the WS model converges to a random graph as $\rho$ reaches 1 . The only graph that yields the smallest value of $L(=1)$ and the largest value of $C(=1)$ is the complete graph. From a Small-World viewpoint, it is necessary to know which network architecture has an absolutely small value of $L$, rather than the order of $L$ (for example, $O(\log N)$ ).

\section{Self-organizing of A SMALl-World Network USING ACO}

Given a complex network with a number of nodes $N$ and an average degree $\langle k\rangle$, one can obtain the average path length $L$ using the Dijkstra method [9] and the clustering coefficient $C$. Then, we propose a self-organizing method for generating a network architecture with a shorter path-length $L$ than that obtained with conventional complex networks, without increasing the average degree $\langle k\rangle$.

A self-organizing method for creating a Small-World inspired from ACO (Ant-Colony Optimization) [8] is described as follows:

(1) Generate a random graph with a number of nodes $N$ and an average degree $\langle k\rangle$. In this case, the generation probability for each link is $p=\langle k\rangle /(N-1)$.

(2) Search for the shortest path from a start node $V_{s}$ to a destination node $V_{d}$ using the Dijkstra method. Pheromones are spread by ants along the shortest path. In the initial stage, the concentration of pheromones is zero independently of links.

(3) Each ant searches for the shortest path, and spreads some amount of pheromones while detecting the higher concentration paths in all combinations of node pairs $V_{s}$ and $V_{d}$. As a result, a difference of pheromone concentration along each path will arise. Namely, the concentration of pheromones will be relatively higher for the paths on which more ants walked than for those on which less ants walked.

(4) Sort the links formed in step (3) on the order of high concentration of pheromones. Then, each vertex in two edges is randomly connected (See Fig.4). This operation is based on the fact that pheromone trails with higher concentration tend to be connected with each other. As the average degree $\langle k\rangle$ increases when one or two edges are newly created, simultaneously cut the links with the order of smaller concentration of pheromones to keep the value of $\langle k\rangle$ as constant as possible.

(5) Calculate the average path-length $L$ and clustering coefficient $C$ for the newly created network in step (4).

(6) If the average path-length $L$ is shorter than a predetermined threshold value $L_{t h}$, stop the operation. Otherwise, return to step (2) and repeat the steps from (2) to (5).

It is very interesting to note that this network architecture obtained through a bottom-up process like ACO is similar to that obtained through an opposite top-down process called the Monte Carlo Bimodal method (See [12]).

\section{Simulation ExPERIMENTS AND RESUlts}
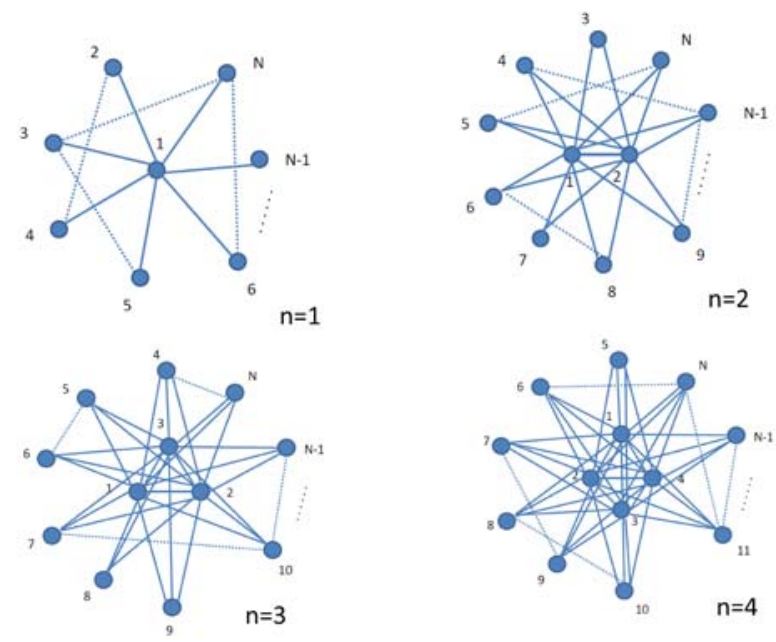

Fig. 6. Multi-star networks with star nodes at the center and their peripheral nodes. The star nodes fully connect with their peripheral nodes, and some peripheral nodes connect with some other peripheral nodes, as denoted by dotted lines. 


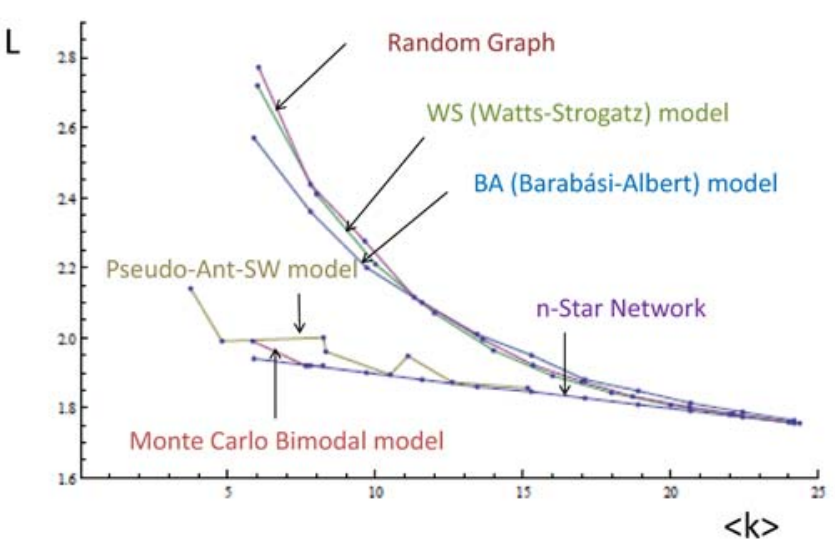

Fig. 7. $L$ vs. $\langle k\rangle$ in several complex networks.

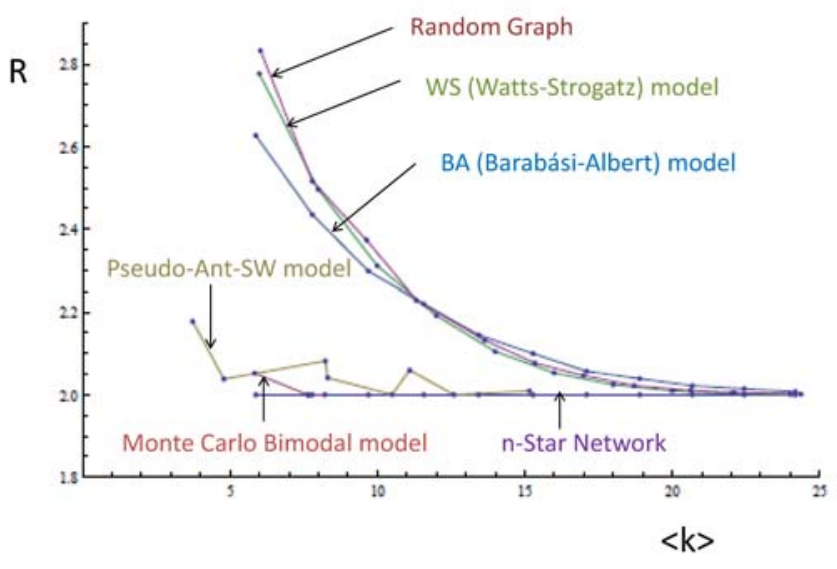

Fig. 8. $R$ vs. $\langle k\rangle$ in several complex networks.

Figure 5 (left) shows the adjacency matrix reflecting pheromone concentration in the Pseudo-Ant-SW method when $N=100$. The thicker the color, the higher the pheromone concentration. Figure 5 (right) shows the multi-star network corresponding to the adjacency matrix in Figure 5 (left) from two different points of view. From these figures, we found that the network architecture consists of a few stars with many links and many peripheral nodes with a few links, and these peripheral nodes are mutually connected with a small probability. Furthermore, from the values of the adjacency matrix and the connection configuration around nodes number 41 and 81, we can find a multi-star (in a sense, a "fractal") structure in the network. This results from the algorithm according to which the higher concentration nodes connect with one another in the Pseudo-Ant-SW method.

For the n-star networks (we can call the multi-star network the $\mathrm{n}$-star network), the average degree $\langle k\rangle$, average path- length $L$ and clustering coefficient $C$ are calculated as follows;

$$
\begin{array}{rr}
\langle k\rangle=\frac{n(2 N-n-1)}{N} ; & n=1,2,3, \ldots, N . \\
L=\frac{2 N(N-n-1)+n(n+1)}{N(N-1)} ; & n=1,2,3, \ldots, N . \\
C=\frac{\frac{n\left\{{ }_{n-1} C_{2}+(n-1)(N-n)\right\}}{N-1} C_{2}}{N}+(N-n) \\
C=0 ; & n=2,3, \ldots, N . \\
& n=1 .
\end{array}
$$

The relationships between $\langle k\rangle$ and $n$ (Eq.(1)), $L$ and $n$
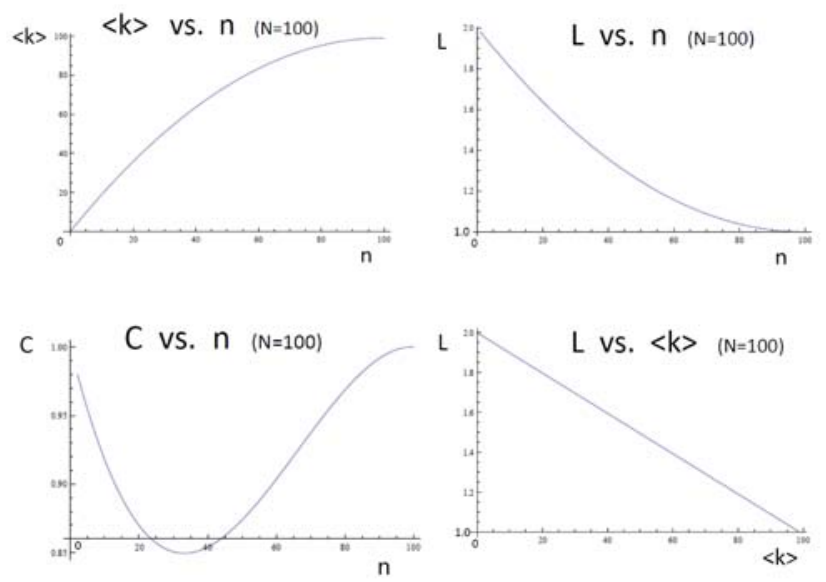

Fig. 9. $\langle k\rangle$ vs. $n$ (upper left), $L$ vs. $n$ (upper right), $C$ vs. $n$ (bottom left), and $L$ vs. $\langle k\rangle$ (bottom right) in multi-star networks with n-star nodes and their peripheral nodes.

(Eq.(2)), and between $C$ and $n$ (Eq.(3)) are shown in Figure 9 , at the upper left, upper right and bottom left of the figure, respectively. For $n=1$, the $\mathrm{n}$-star network becomes a so-called single star network, and the network becomes a complete graph for $n=N-1$ and $N$. The average degree $\langle k\rangle$ monotonically increases from 0 to $N$. On the other hand, $L$ monotonically decreases from 2 to 1 as $n$ increases. The clustering coefficient $C$ decreases once, reaching a minimum value around $\langle k\rangle=33$, and then increases with $n$. If $n$ is removed using Eqs.(1) and (2) to obtain the relationship between $L$ and $\langle k\rangle$, we obtain Eq.(4) as follows:

$$
L=\frac{2 N-\langle k\rangle-2}{N-1}=2-p
$$

The relationship between $L$ and $\langle k\rangle$ for $\mathrm{N}=100$ is shown in Figure 9 (bottom right). Although Eq.(4) is derived for $\langle k\rangle$ values, each value corresponding to $n=1,2,3, . ., N$, it can be shown that this equation is also valid for any intermediate values of $\langle k\rangle$ with arbitrary numbers of peripheral edges connected with each other, which are represented by dotted lines in Figure 6.

Next, we analyze the characteristics of several types of complex networks using Lagrange multipliers. In the case of n-star networks, for a given value of $\langle k\rangle$, we define the $R$ value as follows in order to obtain the minimum value of $L$;

$$
R \equiv L+\lambda\langle k\rangle
$$


By calculating the partial derivative of $R$ with respect to $n$, we obtain $\lambda=1 /(N-1)$. Substituting this formula into Eq.(5), we obtain the invariant $R \equiv 2$, independent of the values of $L$ and $\langle k\rangle$. This means that the $R$ value is invariant for $\mathrm{n}$ star networks. Therefore, we can comparatively evaluate each network as the degree of Small-World (we may call it "SmallWorldness") based on this invariant $R$. The relationships between $L$ and $\langle k\rangle$, and $R$ and $\langle k\rangle$ are shown in Figures 7 and 8 , respectively. The $R$ values are always 2 for n-star networks, as mentioned before. On the other hand, other networks such as the random graph, the WS and BA models have relatively larger $R$ values than 2 . Although the BA model possesses a "scale-free" characteristic with the order of $L \propto O(\log N)$, the $R$ value is not so small compared to that obtained in the random graph and WS model. The proposed Pseudo-Ant-SW and Monte Carlo Bimodal models (See [12] for the latter) have remarkably small values of $L$ and $R$. In particular, the $R$ value in the Monte Carlo Bimodal model almost reaches 2 in n-star networks. This results indicate that these networks achieve the maximum "Small-Worldness" for a given average degree $\langle k\rangle$ (i.e., the minimum value of $L$ with an appropriate average degree $\langle k\rangle$ ) .

\section{APPlying N-STAR NETWORKS TO AIRLINE NETWORKS}

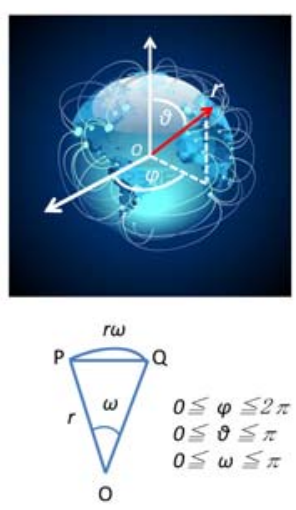

3D Spherical polar coordinate: $(r, \vartheta, \varphi)$. Distance between any two points: $\mathrm{P}\left(r_{1}, \vartheta_{1}, \varphi_{1}\right)$ and $\mathrm{Q}\left(r_{2}, \vartheta_{2}, \varphi_{2}\right)$

$\overline{\mathrm{PQ}}=\sqrt{r^{2}\left(\cos \vartheta_{1}-\cos \vartheta_{2}\right)^{2}+r^{2}\left(\sin \vartheta_{1} \cos \varphi_{1}\right.}$.

$\overline{\left.\sin \vartheta_{2} \cos \varphi_{2}\right)^{2}+r^{2}\left(\sin \vartheta_{1} \sin \varphi_{1}-\sin \vartheta_{2} \sin \varphi_{2}\right)^{2}}$

When the angle between $\overline{O P}$ and $\overline{O Q}$ is $\omega$,

$\cos \omega=\left(2 r^{2}-\overline{P Q^{2}}\right) / 2 r^{2}, \quad \omega=\cos ^{-1}\left(2 r^{2}-\overline{P Q^{2}}\right) / 2 r^{2}$.

Accordingly, the distance $\widehat{P Q}$ on the surface of a sphere is,

$\widehat{P Q}=r \omega=r \cos ^{-1}\left(2 r^{2}-\overline{P Q}\right) / 2 r^{2}$.

Fig. 10. Calculation of the cruising distance in global airline networks.

In the previous sections, we have found that the n-Star network could be formed in a self-organizing way by an ACOinspired Small-World network. An essential reason why the nStar network shows a high degree of "Small-Worldness" is the architecture consisting of its clique with $\mathrm{n}$ star nodes and their peripheral nodes with some mutual links. This advantageous architecture can be applied to real-world problems such as logistics, for example, reorganizing an efficient next-generation airline network, while reflecting the importance ranking of a city, its population and the distance between any two cities in the world. For that purpose, it might be possible to generate from scratch (i.e., a random graph) an n-Star network for the airline network in a self-organizing way while considering the importance ranking of a city etc. However, it is much easier to map an n-Star network onto a global airline network while considering the above importance ranking of a city etc. We will use the latter method in the following section for realizing a global airline network.

It is well known that the current airline network has a "scalefree" characteristic like the BA model (see also Table 1). As is proved in Sec.III, the $L$ value of the n-star network is much smaller than that of the BA model in the range of a small value of $\langle k\rangle$. For example, the average path-length $L$ of the $\mathrm{n}$-star network is $24.4 \%$ smaller than that of the BA model for $\langle k\rangle=5.88$. Very recently, a next-generation airplane, the Boeing 787, has been brought into service [10]. This airplane is middle-sized and has good characteristics that are designed to ensure energy-saving. It can operate a non-stop flight over a distance of 14,200-15,200 km (this distance corresponds to a flight between Tokyo in Japan and Johannesburg in South Africa) because of its light body. The n-star networks could be applied to the design of next-generation global airline networks, as an example of logistics. Figure 10 shows how to calculate the distance between any two cities, $\mathrm{P}$ and $\mathrm{Q}$ on the surface of the Earth in 3D spherical polar coordinates $(r, \theta$, $\phi)$. A British consulting company (GaWC) ranked important cities in the world [11]. We chose the top 100 cities from this list (see Appendix) and compare in the next section the average path-lengths $L$ among five kinds of airline networks. We selected five major cities on five continents from these top cities that occupy a central place in the world in terms of economy and geography: Tokyo, Chicago, Frankfurt, Sydney and Johannesburg.

\section{Simulation Experiments and Results in SEVERAL AIRLINE NETWORKS}

We construct the following five kinds of airline networks based on the n-star networks, and compare their performance in terms of the Small-World, i.e., of efficiency and convenience for both flying and travelers. First, we define the following parameters for constructing several network models:

- Average flight service per city: $\langle k\rangle$.

- Population of city $i: \operatorname{Po}(i)$.

- Spherical distance between city $i$ and city $j$ on the surface of the Earth: $d_{i j}$

- Traffic between city $i$ and city $j: t_{i j} \propto P_{o}(i) \cdot \operatorname{Po}(j)$.

- Importance of relation between city $i$ and city $j$ (weight value): $w_{i j} \propto t_{i j} / d_{i j} \propto P o(i) \cdot \operatorname{Po}(j) / d_{i j}$.

- Physical average distance between any two cities on the surface of the Earth: $L$ (distance).

- $R$ value based on the physical average distance $L$ (distance): $R$ (distance).

- Average number of steps between any two cities: $L$ (steps)

- $R$ value based on the average number of steps $L$ (steps): $R$ (steps).

- Threshold for traffic and importance of relation: Th.

The spherical distance $d_{i j}$ between a city $i$ and a city $j$ is calculated using the equation shown in Fig.10. Traffic $t_{i j}$ between a city $i$ and a city $j$ can be proportional to the 
multiplication of the populations of two cities. The importance of the relation between a city $i$ and a city $j$ can be proportional to the traffic and reversely proportional to the distance between the two cities. Namely, when the traffic is larger, and the distance is shorter, the importance of the relation between two cities becomes higher. We will construct the following five kinds of airline network models based on these parameters.

(1) An airline network with frequent traffic (denoted as "Traffic + peripheral links" in Figures 11-16): This airline network is formed based on the population of cities and their traffic in proportion to the multiplication of the population in each city. As the threshold value $T h$ of traffic changes, the average degree $\langle k\rangle$ changes. Namely, if the traffic between two cities $i$ and $j$ is larger than the threshold value $T h$, the corresponding component $a_{i j}$ of an adjacency matrix a is 1 , otherwise 0 . Therefore, there are some peripheral links between some cities according to the threshold.

(2) An airline network with frequent traffic and city rank (denoted as "Traffic + peripheral links + city rank" in Figures 11-16): This airline network is formed based on the population of cities and their traffic in proportion to the multiplication of the population in each ranked city. Furthermore, the values (i.e., the importance of the relation between two cities) in proportion to each city rank are weighted according to the above evaluation (i.e, $w_{i j} \propto t_{i j} / d_{i j}$ ). Similar to the case of (1), if the importance between two cities is larger than the threshold value $T h$, the corresponding component $a_{i j}$ of an adjacency matrix $\mathbf{a}$ is 1 , otherwise 0 . Therefore, there are some peripheral links between some cities according to the threshold. Note that the top ranked few selected cities (e.g., the top five cities) are not necessarily fully connected by forming a "clique (sub-complete network)" in the cases of (1) and (2).

(3) An airline network with 5 star cities and their peripheral links (denoted as 5 star + peripheral links around the nearest star" in Figures 11-16): This network consists of 5 star cities with their peripheral city links around the nearest star city. As the 5 star cities, we chose Tokyo in Japan, Sydney in Australia, Chicago in the US, Frankfurt in Germany, and Johannesburg in South Africa, for example, because these cities are located near at the center of each continent, and play important roles in terms of geography and/or economics. There are some peripheral links between some cities around each nearest star city. As the number of peripheral cities increases, the average degree $\langle k\rangle$ increases. This network includes a sub-complete network (i.e., a clique) among the above 5 star cities.

(4) An airline network with 5 star cities and their peripheral links at random (denoted as 5 star + peripheral links in Figures 11-16): This network consists of the 5 star cities with their peripheral city links. The peripheral cities are selected at random within the non-stop cruising distance of a Boeing 787 (i.e., 14,200-15,200km). As the number of peripheral cities increases, the average degree $\langle k\rangle$ increases as well.

(5) An airline network with 5 star cities and their peripheral links with city rank (denoted as 5 star + peripheral links+ city rank in Figures 11-16): This network also consists of the 5 star cities with peripheral city links. The peripheral cities connect with one another based on each city weight value $w_{i j}$. The city weight value $w_{i j}$ is defined in proportion to the traffic $t_{i j}$ and in reverse proportion to the distance $d_{i j}$ between two cities.

We calculated the average distance $L$ (distance) between any two cities $i$ and $j$, and show the results in Fig.11. From these values of $L$, we calculated the $R$ (distance) values and show the results in Fig.12. These distances are based on the physical spherical distance on the surface of the Earth. On the other hand, another important issue for airline networks is the number of transits, because the fewer the transits, the more convenient traveling is for passengers. Fig. 13 shows the average number of steps $L(s t e p)$ to reach any destination city from any starting city. If the $L(s t e p)$ is less than 2 , travelers can reach any destination city with only one transit. In this sense, an average small value $L(s t e p)$ is extremely important as well as the average physical cruising distance $L$ (distance), for airline companies, travelers and Earth's environment in terms of reducing fuel consumption, travel time and greenhouse gases such as carbon dioxide. From these values of $L($ steps $)$, we also calculated the values of $R($ steps $)$, and show them in Fig.14 as well. From this figure, we can easily evaluate the "Small-Worldness" for each network, where the networks (4) and (5) have better characteristics in terms of "Small-Worldness", especially in a relative smaller range of $\langle k\rangle$, compared to the other networks (1)-(3).

When considering the clustering coefficients $C$ in Fig.15 and the maximum eigenvalues in Fig.16, the network with 5 star cities and their peripheral links around the nearest star (3) is the smallest among the five kinds of networks, where the values $C$ increase almost in proportion to the increase of $\langle k\rangle$. Networks (1) and (2) produce some relatively larger values. Network (4) yields an intermediate value among the five kinds of networks.

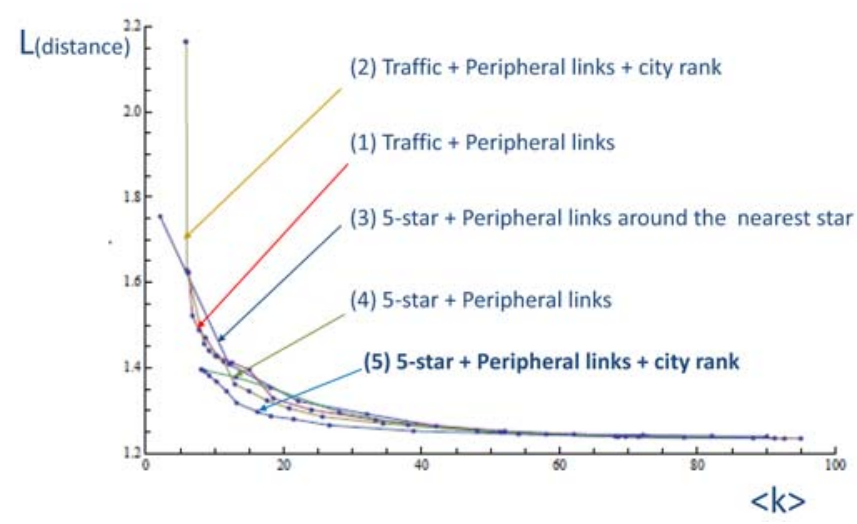

Fig. 11. $L($ distance $)$ versus $\langle k\rangle$ for distance in global airline networks. $L$ is normalized by the radius $r$ of the Earth, $6,356 \mathrm{Km}$ (from the pole to the center) $-6,378 \mathrm{Km}$ (from the equator to the center).

\section{DISCUSSION}

We have re-examined the characteristics of the Small-World (SW) in several complex networks from the viewpoint of the 


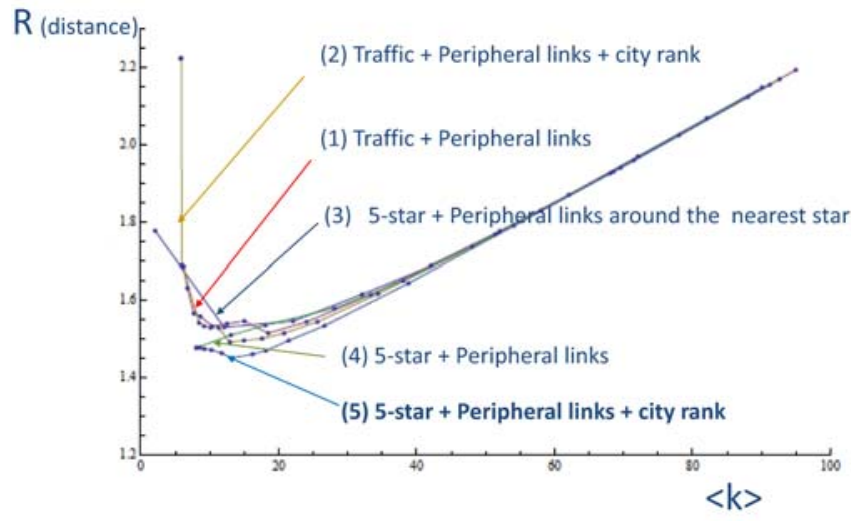

Fig. 12. $R$ (distance) versus $\langle k\rangle$ for distance in global airline networks. $R$ is normalized by the radius $r$ of the Earth, $6,356 \mathrm{Km}$ (from the pole to the center) $-6,378 \mathrm{Km}$ (from the equator to the center).

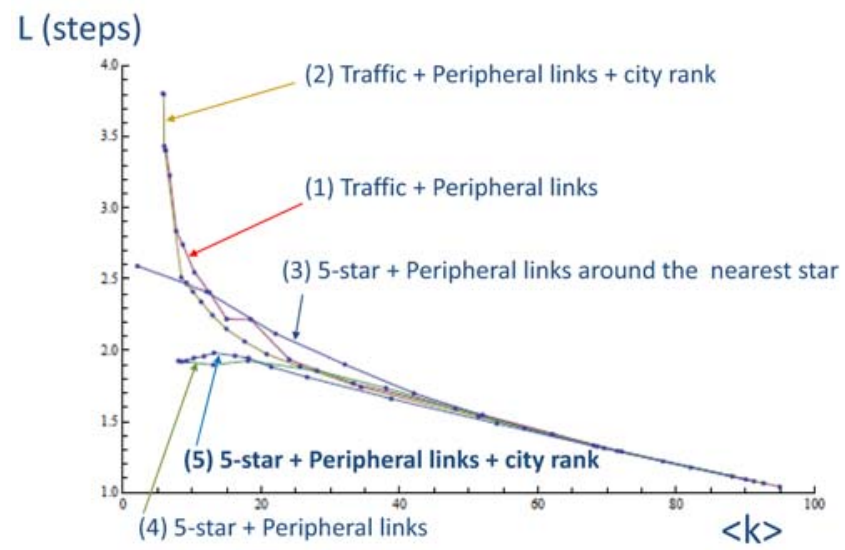

Fig. 13. $L($ steps $)$ versus $\langle k\rangle$ for the number of transits in global airline networks.

"absolute" average path-length, because conventional complex networks known as Small-World are not necessarily SW in a rigorous sense. A method inspired by ACO is proposed to create a new Small-World with the smallest average pathlength $L$. Also, we compared several characteristics in conventional typical complex networks from different viewpoints. In particular, we defined a new evaluation criterion, the $R$ value, in order to minimize the average path-length $L$ when $\langle k\rangle$ is constant. We found that the $R$-value reaches a minimum value and is invariant for the n-star networks. Several kinds of complex networks can be evaluated based on this $R$-value in terms of the Small-World ("Small-Worldness").

On the other hand, based on the above knowledge, we could also design an optimized next-generation airline network, as shown in Fig.17, where 5 star cities are completely connected (i.e., forming a "clique") and their peripheral cities are adequately connected based on demand (i.e., traffic, importance and value of relation between two cities). This network architecture has not only the merit of saving mileage, but also is more efficient and convenient to both airline companies

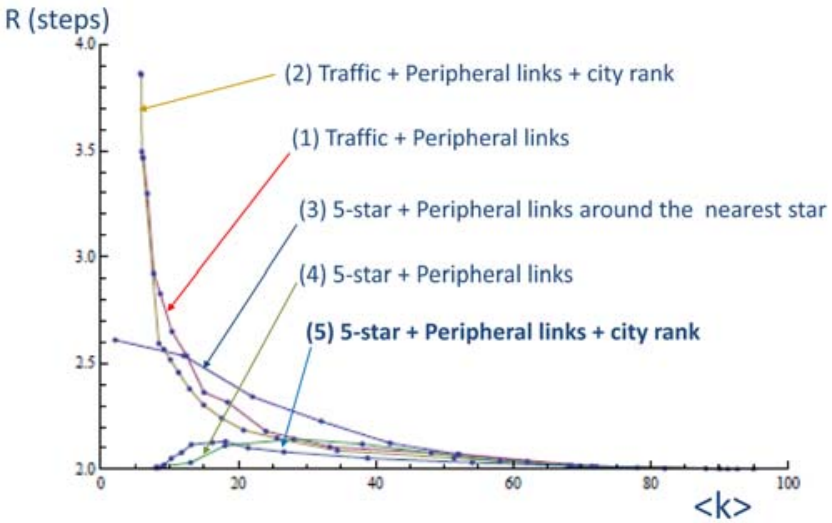

Fig. 14. $R$ (steps) versus $\langle k\rangle$ for the number of transits in global airline networks.

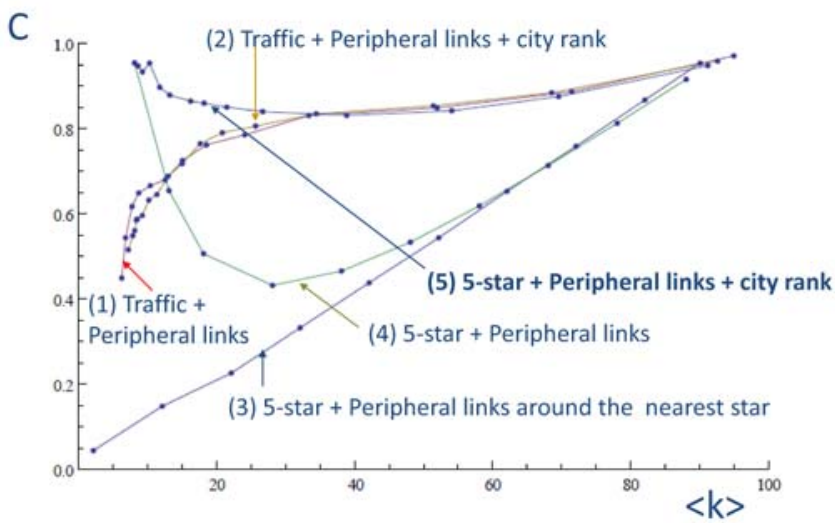

Fig. 15. $C$ versus $\langle k\rangle$ in global airline networks.

and travelers because of the shortest cruising distance (e.g., $1.32 r=8,404 \mathrm{~km}$ for $\langle k\rangle=13.2)$ and smallest number of transits (i.e., only one transit is necessary between any two cities for any value of $\langle k\rangle$ ) on average, which will also have a beneficial impact on Earth's environment in terms of reducing greenhouse gases (e.g., carbon dioxide $\left(\mathrm{CO}_{2}\right)$ ), while enhancing communications and transportation worldwide.

\section{CONCLUSION}

We described a new generation airline network as a realworld application of a novel Small-World (SW) network architecture. An emergent method that creates a new type of SmallWorld network with less average path-length than that obtained with conventional small-world networks was presented. This method is inspired by an Ant-Colony Optimization (ACO) algorithm. The resultant network architecture becomes a multistar network, which yields a large clustering coefficient and the shortest average path-length among the conventional complex networks such as a Watts-Strogatz model and a BarabásiAlbert model etc., from both a theoretical and an experimental analysis of the properties of those networks. Considering the advantageous properties of the multi-star network in real-world 
Max. Eigen value

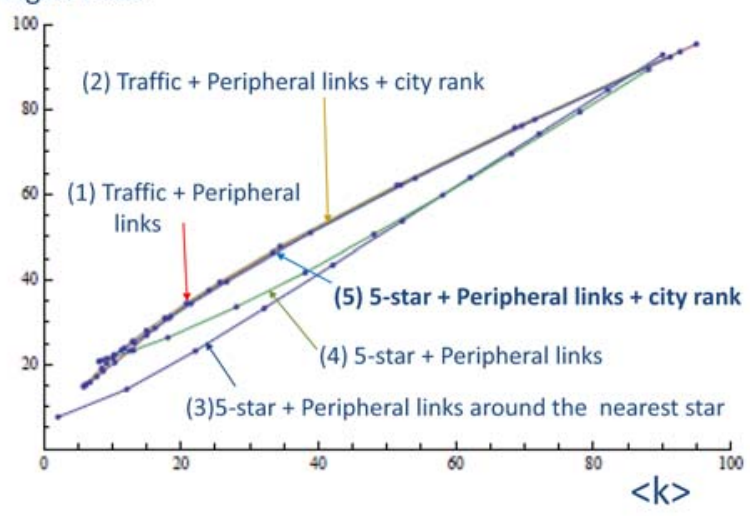

Fig. 16. Eigenvalue versus $\langle k\rangle$ in global airline networks.

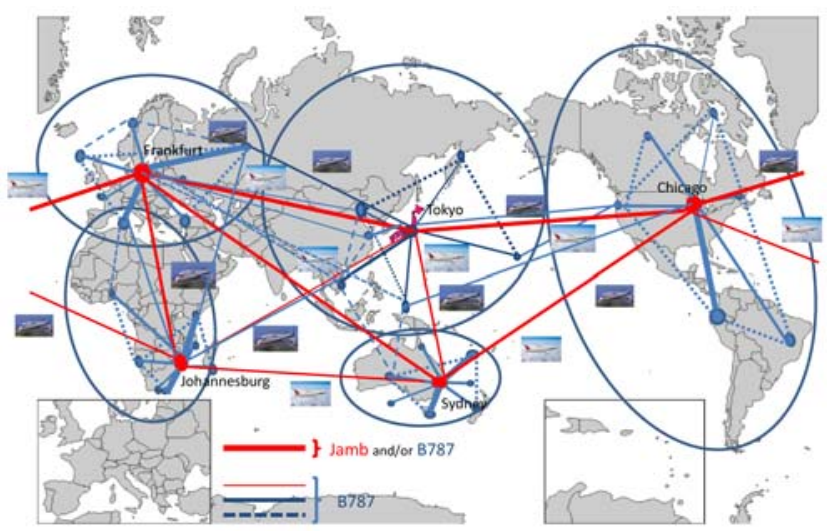

Fig. 17. Schematic diagram of a new generation global airline network in the near future.

applications, it could be used to design a new generation global airline network superseding in terms of efficiency and convenience the current, conventional airline network owing to fewer transits and a shorter cruising distance on average from any starting point to any destination on Earth. This will be beneficial not only both to travelers and airline companies, but will also contribute to the reduction of greenhouse gases such as carbon dioxide $\left(\mathrm{CO}_{2}\right)$ in the near future, while enhancing communications and transportation worldwide..

\section{ACKNOWLEDGEMENT}

The author would like to express his gratitude to Drs. H. Ohsaki, K. Leibnitz, H. Suzuki and F. Peper for fruitful discussions and their useful comments on this study, and to Dr.J-C. Terrillon for his proof-reading this paper with some useful comments.

\section{REFERENCES}

[1] http://www.unitedairlines.co.jp/core/download/UAL_NA_10_09.pdf

[2] http://www.unitedairlines.co.jp/core/download/UAL_World_10_09.pdf

[3] S. Milgram, The Small-World Problem, pp.61-67, Psychology Today, 1967.

[4] J.D. Watts, Small Worlds, The Dynamics of Networks between Order and Randomness, Princeton University Press, 1999.
[5] A.-L. Barábasi, R, Albert, Emergence of Scaling in Random Networks, Science, Vol.286,pp.509-512, 1999.

[6] Yahoo's SW experiment using Facebook, http://www.zdnet.com/blog/facebook/yahoo-facebook-test-8220sixdegrees-of-separation-8221-idea/2678.

[7] N. Masuda, N. Konno, Complex Networks, From Fundamentals to Applications, Kindai Kagaku-sha (in Japanese), 2010.

[8] E. Bonabeau, M. Dorigo, G. Theraulaz, Swarm Intelligence, From Natural to Artificial Systems, Oxford University Press, 1999.

[9] E.W. Dijkstra, A Note on Two Problems in Connexion with Graphs, Numerishe Mathematik 1, pp.269-271, 1959.

[10] Boeing 787 Dream Liner, http://www.newairplane.com/.

[11] J.V. Beaverstock, R.G. Smith, P.J. Taylor, A Roster of World Cities, pp.445-458, vol.15, issue 6, Cities, Dec.1999.

[12] H. Sawai, Emergence and Analysis of A New Small-World Network, Japanese Society for Artificial Intelligence (JSAI) Joint-Technical Meeting and Symposium, Tokyo, Dec.2011. (in Japanese)

\section{APPENDIX}

Table 2. Top 100 cities (shown in part) in the world. [11] The population is represented in units of ten thousands.

\begin{tabular}{|c|c|c|c|c|}
\hline No. & Rank & City & Country & Pop. \\
\hline 1 & $\overline{\mathrm{A}++}$ & London & 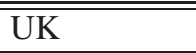 & 1250 \\
\hline 2 & $\mathrm{~A}++$ & New York & US & 800 \\
\hline 3 & $\mathrm{~A}+$ & Hong Kong & China & 700 \\
\hline 4 & $\mathrm{~A}+$ & Paris & France & 1184 \\
\hline 5 & $\mathrm{~A}+$ & Singapore & Singapore & 507 \\
\hline 6 & $\mathrm{~A}+$ & Tokyo & Japan & 1320 \\
\hline 7 & $\mathrm{~A}+$ & Shanghai & China & 2300 \\
\hline 8 & $\mathrm{~A}+$ & Chicago & US & 300 \\
\hline 9 & $\mathrm{~A}+$ & Dubai & UAE & 226 \\
\hline 10 & $\mathrm{~A}+$ & Sydney & Australia & 434 \\
\hline .. & .. & ........ & $\ldots .$. & $\ldots$ \\
\hline .. & .. & $\ldots \ldots$. & $\ldots .$. & $\ldots$ \\
\hline 44 & A- & Santiago & Chili & 467 \\
\hline 45 & A- & Lisbon & Portugal & 565 \\
\hline 46 & A- & Philadelphia & US & 580 \\
\hline 47 & A- & Johannesburg & South Africa & 388 \\
\hline 48 & $\mathrm{~B}+$ & Dusseldorf & Germany & 58 \\
\hline 49 & $\mathrm{~B}+$ & Stockholm & Sweden & 75 \\
\hline 50 & $\mathrm{~B}+$ & Prague & Czech & 120 \\
\hline 51 & $\mathrm{~B}+$ & Montreal & Canada & 162 \\
\hline 52 & $\mathrm{~B}+$ & Roma & Italy & 272 \\
\hline 53 & $\mathrm{~B}+$ & Hamburg & Germany & 174 \\
\hline 54 & $\mathrm{~B}+$ & Manila & Philippine & 166 \\
\hline .. & .. & ........ & $\ldots .$. & $\ldots$ \\
\hline .. & .. & ...... & $\ldots .$. & $\ldots$ \\
\hline 91 & B- & Panama & Panama & 71 \\
\hline 92 & B- & Helsinki & Finland & 59 \\
\hline 93 & B- & Shenzhen & China & 1200 \\
\hline 94 & B- & Guatemala City & Guatemala & 101 \\
\hline 95 & B- & Stuttgart & Germany & 60 \\
\hline 96 & B- & Abu Dhabi & UAE & 143 \\
\hline 97 & B- & Birmingham & UK & 100 \\
\hline 98 & B- & Kolkata & India & 458 \\
\hline 99 & B- & Koln & Germany & 100 \\
\hline 100 & B- & St. Louis & US & 283 \\
\hline
\end{tabular}

